FOLIA

Amazónica

Revista del Instituto de Investigaciones

de la Amazonía Peruana

\title{
PLANTAS USADAS PARA COMBATIR LA PANDEMIA DEL COVID-19 EN UNA COMUNIDAD INDÍGENA URARINA DEL DEPARTAMENTO DE LORETO, PERÚ
}

\author{
Margarita DEL AGUILA VILLACORTA ${ }^{1, *}$; Manuel MARTÍN BRAÑAS ${ }^{2}$; \\ Emanuele FABIANO ${ }^{3}$; Ricardo ZÁRATE GÓMEZ2; Juan José PALACIOS VEGA²; \\ Samuel NURIBE ARAHUATA*; Wendy Darlene MOZOMBITE RUÍZ ${ }^{2}$
}

1 Universidad Nacional de la Amazonía Peruana - UNAP. Iquitos, Perú.

2 Instituto de Investigaciones de la Amazonía Peruana - IIAP. Iquitos, Perú.

3 Pontificia Universidad Católica del Perú - PUCP. Lima, Perú.

${ }^{4}$ Comunidad Nativa de Nueva Unión. Loreto, Perú.

* Correo electrónico: madelavi1494@gmail.com

\section{RESUMEN}

El artículo presenta las plantas usadas para combatir la pandemia del COVID-19 en una comunidad del pueblo indígena urarina, en la región Loreto, Perú. Se realizaron entrevistas semi estructuradas a promotores de salud, sabios, sabias, dirigentes y docentes de la comunidad durante la primera y segunda ola de la pandemia, de mayo del 2020 a junio del 2021. Al no poderse realizar viajes a la comunidad durante la primera ola de contagios, las entrevistas fueron realizadas vía telefónica o a través de aplicaciones de mensajería como WhatsApp o Messenger. Durante la segunda ola se realizó trabajo de campo, complementando la información con entrevistas directas a pobladores de la comunidad y grupos focales sobre el uso de las plantas. Se realizó la colecta de las plantas usadas de los lugares identificados en las entrevistas y en los diferentes grupos focales. Se identificaron 16 especies vegetales usadas específicamente en la pandemia. Cuatro de ellas fueron introducidas en América a partir del siglo XVI y son usadas comúnmente de manera integrada con especies que son de uso tradicional en la comunidad. Se identificaron también cuatro especies que han sido usadas para fortalecer el cuerpo y el espíritu frente a la enfermedad del COVID-19.

PALABRAS CLAVE: Medicina tradicional, plantas medicinales, enfermedades respiratorias. 


\title{
PLANTS USED TO COMBAT THE COVID-19 PANDEMIC IN AN INDIGENOUS URARINA COMMUNITY IN THE DEPARTMENT OF LORETO, PERU
}

\begin{abstract}
We report the plants used to combat the COVID-19 pandemic in a community of the Urarina indigenous people, in Loreto, Peru. Semi-structured interviews were conducted with health promoters, wise men, leaders and teachers of the community during the first and second waves of the pandemic, from May 2020 to June 2021. It was not possible to make trips to the community during the first wave of infections, so the interviews were carried out by telephone or through messaging applications such as WhatsApp or Messenger. During the second wave, field work was carried out, complementing the information with direct interviews with community residents and focus groups about the use of the plants. The plants used were collected from the places identified in the interviews and in the different focus groups. 16 plant species used specifically in the pandemic were identified. Four of them were introduced in America from the 16th century and are commonly used in an integrated way with species that are traditionally used in the community. Four species were also identified that have been used to strengthen the body and spirit against the COVID-19 disease.

KEYWORDS: Tradicional medicine, medicinal plants, respiratory diseases.
\end{abstract}




\section{INTRODUCCIÓN}

Las epidemias llegaron a la Amazonía cuando los primeros europeos iniciaron la conquista de sus territorios en el siglo XVI (Crosby, 1972; Cordero, 2001), no obstante, fue a partir del siglo XVII, con la llegada de los misioneros jesuitas y franciscanos a la Amazonía y la instalación de las primeras misiones y reducciones, cuando las epidemias empezaron a impactar de manera notable en la población indígena amazónica (Denevan, 1992; Ludescher, 2001). Desde entonces, las epidemias han sido más frecuentes en las comunidades indígenas. En pleno siglo XX, epidemias como el sarampión, la viruela, el cólera o la influenza golpearon a los pueblos indígenas amazónicos, causando muerte y desolación en sus comunidades. Muchos pueblos originarios desaparecieron completamente debido a las epidemias, la mayoría durante los primeros doscientos años después de la conquista, otros pudieron aplicar estrategias de alejamiento y conocimientos tradicionales para minimizar sus impactos y sobrevivir hasta nuestros días.

La pandemia del COVID-19 ha puesto a prueba los sistemas de conocimiento de cada uno de los 51 pueblos amazónicos originarios que hoy habitan en la Amazonía peruana. Cuando el virus llegó a sus comunidades, pocos eran los que creían que los pueblos indígenas serían capaces de enfrentar una enfermedad que escapaba del control de la medicina occidental y que estaba provocando una mortalidad elevada en los llamados países desarrollados. La precaria atención de los sistemas de salud y la lejanía de algunas comunidades, hacía esperar una falta de abastecimiento de medicinas y oxígeno para las personas enfermas y, por lo tanto, una mortalidad elevada, no obstante, el impacto fue menor al esperado, alcanzando en setiembre del 2021 una tasa de letalidad (fallecidos frente a confirmados) del 1,34\% (Organización Panamericana de la
Salud, 2021), una cifra mucho menor al 9,4\% sincerado en el mismo periodo para la población no indígena en el Perú (MINSA, 2021). La aplicación de los conocimientos tradicionales sobre las especies de plantas con propiedades curativas puede haber sido un factor favorable para que la tasa de letalidad no haya alcanzado porcentajes mayores.

Uno de los pueblos que ha enfrentado la pandemia usando los conocimientos existentes sobre el uso de las plantas que curan, ha sido el urarina. El pueblo urarina se asienta sobre los territorios bañados por el río Chambira y sus tributarios. Con una población aproximada de 5000 personas, hoy en día afronta nuevas dinámicas socio económicas que lo hacen más vulnerable, debido a las relaciones no siempre horizontales que establece con diferentes actores de la sociedad nacional. La historia del pueblo urarina está marcada por la época del patronazgo durante el boom del caucho y la subsiguiente extracción de diversos productos del bosque. Como otros pueblos amazónicos no han podido evitar el azote de diversas epidemias que llegaron a la región, como el cólera, el sarampión o la viruela. La llegada del COVID-19 ha supuesto una amenaza mucho mayor, por la agresividad de la enfermedad y por el relacionamiento mucho más cercano con diferentes actores de la sociedad nacional, lo que ha favorecido los contagios externos. El presente artículo presenta las especies vegetales usadas por una comunidad urarina para combatir la pandemia del COVID-19.

\section{MATERIALES Y MÉTODOS}

El área de estudio se localizó en la comunidad urarina de Nueva Unión, ubicada en la cuenca del río Chambira. El pueblo urarina, cuya lengua es la única representante de la familia lingüística shimaco, habita en los territorios bañados por 

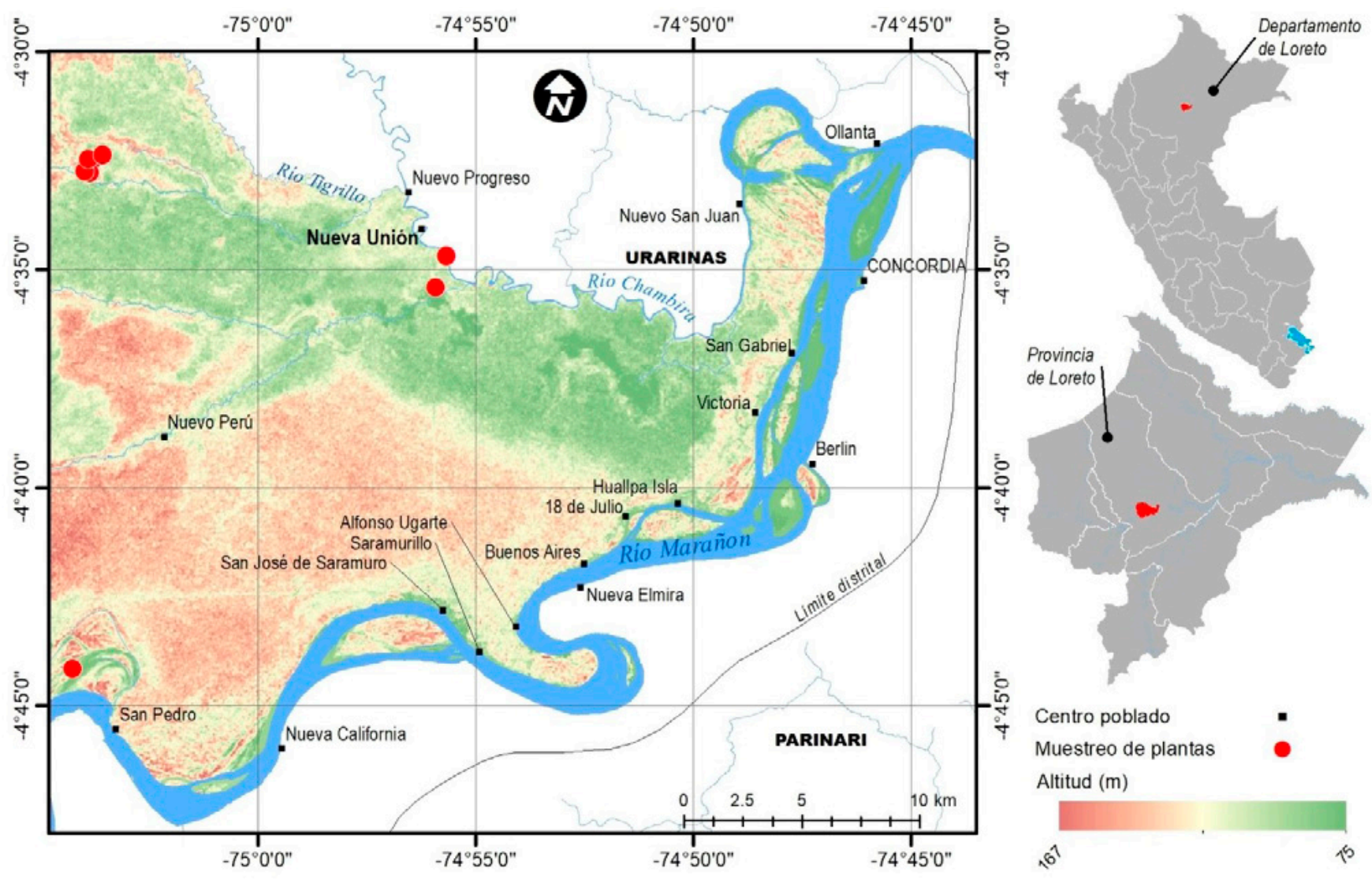

Figura 1: Mapa de ubicación de la zona de estudio.

el río Chambira y sus tributarios y tiene una población aproximada de 5000 personas. La comunidad Nueva Unión pertenece al distrito Urarinas, provincia Loreto, departamento Loreto, Perú, ubicándose en las siguientes coordenadas geográficas $4^{\circ} 34^{\prime} 32,34$ "latitud Sur y $74^{\circ} 55^{\prime} 58,02 "$ longitud Oeste, con una altitud que varía entre los 75 y 170 metros sobre el nivel del mar (Figura 1), con predominancia de relieves de terrazas bajas y medias con drenajes que van desde bueno a imperfecto. Para acceder a la comunidad se debe realizar un viaje por vía terrestre desde la ciudad de Iquitos hasta la ciudad de Nauta. El tiempo que se invierte por carretera es de una hora con 30 minutos. Una vez en Nauta, se debe realizar un viaje por vía fluvial desde Nauta al centro poblado de Ollanta. El viaje fluvial se extiende por siete a ocho horas en un motor de 60 caballos de fuerza. De Ollanta hasta Nueva Unión se viaja por el río Chambira durante una hora con el mismo motor. La comunidad de Nueva Unión presenta una población de 101 habitantes (INEI, 2017). La investigación cumplió con lo establecido en las leyes de protección de los conocimientos colectivos y consulta previa vigentes en el Perú.

El estudio se desarrolló durante el periodo que va de mayo del año 2020 hasta junio del año 2021, en pleno estado de emergencia declarado por el Estado peruano para disminuir el riesgo de contagio por COVID-19. Por este motivo, la investigación exploratoria, en una primera etapa, utilizó como principales métodos cualitativos las entrevistas semiestructuradas realizadas vía telefónica o a través de aplicaciones de mensajería instantánea como WhatsApp y Messenger. En 
una segunda etapa, ya durante la segunda ola de contagios, se pudo realizar una visita de doce días a la comunidad, ampliando el universo de entrevistados y desarrollando grupos focales de identificación y colectando datos de ubicación geográfica mediante el uso de un dispositivo GPS. En total se realizaron 08 entrevistas virtuales en la primera ola de la pandemia y 10 entrevistas directas y 02 grupos focales en la segunda ola. Los grupos focales se desarrollaron con 16 varones adultos y 21 mujeres adultas de la comunidad. La muestra de edades de los entrevistados osciló entre los 20 y los 70 años. Las entrevistas telefónicas fueron grabadas bien usando la aplicación CallApp o a través de los mensajes de voz de las aplicaciones WhatsApp y Messenger; las entrevistas presenciales fueron grabadas digitalmente y transcritas posteriormente para su análisis. Se realizó la colecta (Tabla 1) de las especies medicinales usadas en la comunidad para combatir la pandemia, previamente identificadas en los grupos focales y en las entrevistas semiestructuradas. La colecta se realizó en los lugares donde son aprovechadas habitualmente. La herborización se realizó siguiendo las recomendaciones de Judd et al. (1999). Las muestras fueron depositadas en el Herbario Herrerense $(\mathrm{HH})$ del Instituto de Investigaciones de la Amazonía Peruana. La identificación de las especies fue realizada con la ayuda de guías de identificación (Vásquez, 1997; Ribeiro et al., 1999) y realizando una comparación con las especies clasificadas en el Herbario Herrerense.

\section{RESULTADOS}

Se identificaron 16 especies vegetales que han sido usadas en la comunidad urarina de Nueva Unión para combatir la pandemia del COVID-19 (Tabla 1). La mayoría de estas especies forman parte del vademécum tradicional del pueblo urarina y han sido utilizadas históricamente de manera recurrente para combatir las enfermedades respiratorias y los síntomas relacionados. Cuatro de las especies no son originarias de América, introducidas tardíamente durante la época de la conquista, siendo adoptadas progresivamente y propagadas muy rápidamente en las comunidades amazónicas. Dos de las especies nativas identificadas, el toé y el cordoncillo, han sido usadas de manera más intensa y específica para combatir el COVID-19. Todas las especies menos una son aprovechadas dentro del territorio comunal.

Las especies vegetales usadas pueden ser agrupadas en dos categorías que nos permiten diferenciarlas de acuerdo a su uso específico: plantas usadas para prevenir o combatir los síntomas provocados por el COVID-19 y plantas usadas para sanar o fortalecer el espíritu frente al COVID-19 y otras enfermedades. Dos de las especies identificadas son mixtas y forman parte de ambas categorías, siendo usadas para uno u otro fin.

En el primer grupo se encuentran el ajengibre (biri künai) Zingiber officinale Roscoe, la toronja (turuunjua) Citrus sp., el ajo sacha (kuü) Mansoa alliacea (Lam.) A. H. Gentry, la lancetilla (raansata) Alternanthera brasiliana (L.) Kuntze., el cordoncillo (asiiatüüi) Piper sp., la uña de gato (nekuüüri enuuene) Uncaria guianensis (Aubl.) Gmel, el limón (remuun) Citrus sp., el remo caspi (anuina) Aspidosperma sp., la ishanga (aküsa) Urera sp., el algodón (mishu üjüe) Gossypium barbadense L., la malva (maruba) Malachra alceifolia Jacq., y la guayaba (shabeentu) Psidium guajava L. En el segundo grupo se encuentran el piri piri (biri) Cyperus sp. y la ayahuasca (inunu) Banisteriopsis sp. La mucura (kacha nuta nukuaera) Petiveria alliacea (Lam.) A.H.Gentry y el toé (akaa) Brugmansia suaveolens (Humb. \& Bonpl. ex Willd.) Bercht. \& J.Presl son 
Tabla 1: Datos de colecta de las especies utilizadas para combatir el COVID-19 y sanar el cuerpo y el espíritu por la comunidad de Nueva Unión, Loreto.

\begin{tabular}{|c|c|c|c|c|c|c|c|c|c|}
\hline $\begin{array}{l}\text { Código } \\
\text { de } \\
\text { colecta }\end{array}$ & Familia & Especie & $\begin{array}{l}\text { Nombre } \\
\text { urarina }\end{array}$ & Hábito & $\begin{array}{l}\text { Altitud } \\
\text { (m) }\end{array}$ & $\begin{array}{c}\text { Este (m) } \\
\text { Zona } \\
18 S\end{array}$ & $\begin{array}{l}\text { Norte }(\mathrm{m}) \\
\text { Zona } 18 \mathrm{~S}\end{array}$ & $\begin{array}{l}\text { Zona } \\
\text { UTM }\end{array}$ & Fecha \\
\hline DA 001 & Malvaceae & $\begin{array}{c}\text { Gossypium } \\
\text { barbadense L. }\end{array}$ & Mishu üjüe & Arbusto & 105 & 492169 & 9476500 & 18 Sur & $16 / 06 / 21$ \\
\hline DA 002 & Urticaceae & Urera sp. & Aküsa & Arbusto & 111 & 492756 & 9497653 & 18 Sur & $16 / 06 / 21$ \\
\hline DA 003 & Malvaceae & $\begin{array}{c}\text { Malachra } \\
\text { alceifolia Jacq. }\end{array}$ & Maruba & Sufrútice & 111 & 492754 & 9497652 & 18 Sur & $16 / 06 / 21$ \\
\hline DA 004 & Bignoniaceae & $\begin{array}{c}\text { Mansoa } \\
\text { alliacea (Lam.) } \\
\text { A.H.Gentry }\end{array}$ & Kü̈ & Bejuco & 112 & 492827 & 9497623 & 18 Sur & $16 / 06 / 21$ \\
\hline DA 005 & Amaranthaceae & $\begin{array}{c}\text { Alternanthera } \\
\text { brasiliana (L.) } \\
\text { Kuntze }\end{array}$ & Raansata & Hierba & 114 & 492733 & 9497681 & 18 Sur & $16 / 06 / 21$ \\
\hline DA 006 & Rutaceae & Citrus sp. 1 & Remuun & Arbusto & 116 & 492709 & 9497690 & 18 Sur & $16 / 06 / 21$ \\
\hline DA 007 & Solanaceae & $\begin{array}{c}\text { Brugmansia } \\
\text { suaveolens } \\
\text { (Humb. \& } \\
\text { Bonpl. ex } \\
\text { Willd.) Bercht. } \\
\text { \& J.Presl }\end{array}$ & Akaa & Arbusto & 112 & 492830 & 9497736 & 18 Sur & $16 / 06 / 21$ \\
\hline DA 008 & Piperaceae & Piper sp. & Asiiatüüi & Arbusto & 115 & 492717 & 9497688 & 18 Sur & $16 / 06 / 21$ \\
\hline DA 009 & Rubiaceae & $\begin{array}{c}\text { Uncaria } \\
\text { guianensis } \\
\text { (Aubl.) } \\
\text { J.F.Gmel. }\end{array}$ & $\begin{array}{l}\text { Nekuüüri } \\
\text { enuuene }\end{array}$ & Bejuco & 118 & 508023 & 9493973 & 18 Sur & $16 / 06 / 21$ \\
\hline $\begin{array}{l}\text { Obs. } \\
\text { campo }\end{array}$ & Apocynaceae & $\begin{array}{c}\text { Aspidosperma } \\
\text { sp. }\end{array}$ & Anuina & Arbol & 106 & 507574 & 9492627 & 18 Sur & $17 / 06 / 21$ \\
\hline DA 010 & Zingiberaceae & $\begin{array}{l}\text { Zingiber } \\
\text { officinale } \\
\text { Roscoe }\end{array}$ & Biri künai & $\begin{array}{l}\text { Hierba } \\
\text { rizomatosa }\end{array}$ & 112 & 492768 & 9497789 & 18 Sur & $18 / 06 / 21$ \\
\hline $\begin{array}{l}\text { Obs. } \\
\text { campo }\end{array}$ & Myrtaceae & $\begin{array}{l}\text { Psidium } \\
\text { guajava L. }\end{array}$ & Shabeentu & Arbusto & 119 & 492911 & 9497872 & 18 Sur & $18 / 06 / 21$ \\
\hline $\begin{array}{l}\text { Obs. } \\
\text { campo }\end{array}$ & Rutaceae & Citrus sp. 2 & Turuunjua & Arbusto & 120 & 492877 & 9497472 & 18 Sur & $18 / 06 / 21$ \\
\hline $\begin{array}{l}\text { Obs. } \\
\text { campo }\end{array}$ & Cyperaceae & Cyperus sp. & Biri & Hierba & 121 & 492682 & 9497526 & 18 Sur & $18 / 06 / 21$ \\
\hline $\begin{array}{l}\text { Obs. } \\
\text { campo }\end{array}$ & Malpighiaceae & $\begin{array}{c}\text { Banisteriopsis } \\
\text { sp. }\end{array}$ & Inunu & Bejuco & 114 & 492819 & 9498064 & 18 Sur & $18 / 06 / 21$ \\
\hline $\begin{array}{l}\text { Obs. } \\
\text { campo }\end{array}$ & Phytolaccaceae & $\begin{array}{l}\text { Petiveria } \\
\text { alliacea L. }\end{array}$ & $\begin{array}{l}\text { Kacha nuta } \\
\text { nukuaera }\end{array}$ & Hierba & 117 & 493448 & 9498230 & 18 Sur & $18 / 06 / 21$ \\
\hline
\end{tabular}


Tabla 2: Datos de uso de las especies vegetales identificadas en la comunidad Nueva Unión, Loreto.

\begin{tabular}{|c|c|c|c|c|}
\hline Especie & $\begin{array}{l}\text { Nombre } \\
\text { urarina }\end{array}$ & $\begin{array}{l}\text { Parte } \\
\text { usada }\end{array}$ & Indicación & Uso \\
\hline $\begin{array}{l}\text { Gossypium } \\
\text { barbadense }\end{array}$ & Mishu üjüe & Semilla & $\begin{array}{l}\text { Inflamación de } \\
\text { bronquios. } \\
\text { Protección } \\
\text { de los niños } \\
\text { contra la gripe. }\end{array}$ & $\begin{array}{l}\text { Vía oral: Las semillas tostadas son molidas y mezcladas } \\
\text { con orina de un niño o una niña (dependiendo del } \\
\text { género del enfermo). Se toma tres veces por día. } \\
\text { Con las semillas se elaboran collares que protegen a } \\
\text { los niños contra la gripe. }\end{array}$ \\
\hline Urera sp. & Aküsa & Hoja & Dolor & Vía tópica: Se golpea la parte adolorida con las hojas. \\
\hline $\begin{array}{l}\text { Malachra } \\
\text { alceifolia }\end{array}$ & Maruba & Hoja & $\begin{array}{l}\text { Dolor de } \\
\text { cabeza y fiebre }\end{array}$ & $\begin{array}{c}\text { Vía tópica: Se maceran las hojas en agua y se amasan } \\
\text { hasta que suelten la savia. Se combina con hojas de } \\
\text { limón y lancetilla. El macerado se frota en la cabeza } \\
\text { para aplacar el dolor y la fiebre }\end{array}$ \\
\hline $\begin{array}{l}\text { Mansoa } \\
\text { alliacea }\end{array}$ & Kuӥ & Hoja & $\begin{array}{l}\text { Gripe, } \\
\text { problemas } \\
\text { respiratorios }\end{array}$ & $\begin{array}{l}\text { Vía oral: ILas hojas se cocinan en agua y se toma } \\
\text { caliente o como agua del tiempo. Vía tópica: Las hojas } \\
\text { se queman para ahuyentar la enfermedad. }\end{array}$ \\
\hline $\begin{array}{l}\text { Alternanthera } \\
\text { brasiliana }\end{array}$ & Raansata & Hoja & 'Gripe, fiebre & $\begin{array}{c}\text { Vía tópica: Se realizan baños corporales con la infusión } \\
\text { de las hojas. }\end{array}$ \\
\hline Citrus sp. 1 & Remuun & Hoja & Fiebre & $\begin{array}{c}\text { Vía tópica: Las hojas se mezclan con las de la malva y } \\
\text { se amasan en agua. Se aplica con paños en la cabeza. } \\
\text { El jugo se usa con la hoja del cordoncillo. }\end{array}$ \\
\hline $\begin{array}{l}\text { Brugmansia } \\
\text { suaveolens }\end{array}$ & Akaa & Tallo & $\begin{array}{l}\text { COVID-19, } \\
\text { Dolor de } \\
\text { cuerpo, fiebre. } \\
\text { También como } \\
\text { protección. }\end{array}$ & $\begin{array}{l}\text { Vía tópica: Se machaca el tallo y el jugo resultante se } \\
\text { frota en la parte adolorida. Vía oral: Se hierve el tallo } \\
\text { en agua y se toma una cucharadita todos los días }\end{array}$ \\
\hline Piper sp. & Asiiatüüi & Hoja & $\begin{array}{l}\text { COVID-19, } \\
\text { llagas. }\end{array}$ & $\begin{array}{c}\text { Vía oral: Se hierven las hojas y se mezclan con jugo } \\
\text { de limón. Se toma medio vaso por día. Vía tópica: El } \\
\text { líquido se puede aplicar en llagas provocadas por la } \\
\text { fiebre. }\end{array}$ \\
\hline $\begin{array}{l}\text { Uncaria } \\
\text { guianensis }\end{array}$ & $\begin{array}{l}\text { Nekuüüri } \\
\text { enuuene }\end{array}$ & $\begin{array}{c}\text { Tallo } \\
\text { (corteza) }\end{array}$ & $\begin{array}{l}\text { Dolor de } \\
\text { pecho, } \\
\text { espalda, } \\
\text { desinflamar } \\
\text { pulmones }\end{array}$ & $\begin{array}{l}\text { Vía oral: Se cocina la corteza en agua y se ingiere } \\
\text { como agua del tiempo. }\end{array}$ \\
\hline $\begin{array}{l}\text { Aspidosperma } \\
\text { sp. }\end{array}$ & Anuina & $\begin{array}{c}\text { Tallo } \\
\text { (corteza) }\end{array}$ & Fiebre & $\begin{array}{l}\text { Vía oral: Se cocina la corteza en agua y se ingiere } \\
\text { como agua del tiempo. }\end{array}$ \\
\hline $\begin{array}{l}\text { Zingiber } \\
\text { officinale }\end{array}$ & Biri künai & Raíz & $\begin{array}{l}\text { Gripe, dolor } \\
\text { de garganta, } \\
\text { cabeza o } \\
\text { cuerpo. }\end{array}$ & $\begin{array}{l}\text { Vía tópica: La raíz se machaca en agua y se toma el } \\
\text { jugo. Se mastica un pedazo para quitar el dolor de } \\
\text { cabeza y cuerpo. }\end{array}$ \\
\hline $\begin{array}{l}\text { Psidium } \\
\text { guayava }\end{array}$ & Shabeentu & Hoja & $\begin{array}{l}\text { Còlicos y } \\
\text { diarrea }\end{array}$ & $\begin{array}{c}\text { Vía oral: Se hierven las hojas jóvenes hasta que } \\
\text { el líquido quedé de color negro. Se toma una } \\
\text { cucharadita por día. }\end{array}$ \\
\hline
\end{tabular}




\begin{tabular}{|c|c|c|c|c|}
\hline Citrus sp. 2 & Turuunjua & $\begin{array}{l}\text { Hoja, } \\
\text { fruto }\end{array}$ & $\begin{array}{l}\text { Resfríos, } \\
\text { problemas } \\
\text { respiratorios. }\end{array}$ & $\begin{array}{c}\text { Vía oral: El jugo del fruto se calienta con un poco de } \\
\text { sal y se toma dos o tres veces al día. Se puede tomar } \\
\text { la infusión de las hojas. }\end{array}$ \\
\hline Ciperus sp. & Biri & Hoja & $\begin{array}{l}\text { Proteger o } \\
\text { ahuyentar } \\
\text { enfermedades }\end{array}$ & $\begin{array}{l}\text { Vía tópica: Los bulbos se muelen con poca agua. Se } \\
\text { realizan frotaciones en todo el cuerpo. }\end{array}$ \\
\hline $\begin{array}{l}\text { Banisteriopsis } \\
\text { sp. }\end{array}$ & Inunu & $\begin{array}{c}\text { Tallo } \\
\text { (corteza) }\end{array}$ & $\begin{array}{l}\text { Proteger o } \\
\text { ahuyentar } \\
\text { enfermedades }\end{array}$ & $\begin{array}{l}\text { Vía oral: Se extrae la corteza y se cocina, se toma de } \\
\text { acuerdo a indicaciones del vegetalista. }\end{array}$ \\
\hline $\begin{array}{l}\text { Petiveria } \\
\text { alliacea }\end{array}$ & $\begin{array}{l}\text { Kacha nuta } \\
\text { nukuaera }\end{array}$ & Hoja & $\begin{array}{l}\text { Fiebre, gripe. } \\
\text { También como } \\
\text { protección. }\end{array}$ & $\begin{array}{l}\text { Vía oral: sSe hierven las hojas en agua y se toma el } \\
\text { líquido, lo que sobra se usa en baños. Vía tópica: Las } \\
\text { hojas se queman para ahuyentar la enfermedad. El } \\
\text { humo es inhalado como protección de la enfermedad. }\end{array}$ \\
\hline
\end{tabular}

especies usadas tanto para tratar los síntomas del COVID-19, como para fortalecer el cuerpo y el espíritu frente al COVID-19.

En orden de importancia las partes más usadas de las especies vegetales han sido las hojas, las cortezas, los tallos, la raíz, la semilla y los frutos (Tabla 2). El extracto de las hojas ha sido tomado, tanto al fresco, macerando y amasando las hojas en agua, como en infusiones calientes; las hojas también han sido usadas vía tópica en emplastos o vía nasal a través de inhalaciones del humo al ser quemadas. Las cortezas se han cocinado en agua y el extracto ha sido ingerido como agua del tiempo. La única raíz usada ha sido la del ajengibre, machacándola en agua y tomando el jugo o masticando un pedazo de la misma. Las semillas del algodón ha sido tostada y molida y mezclada con la orina de niños o niñas. Del fruto de la toronja y del limón se ha extraído el jugo, en el primer caso se ha calentado con un poco de sal y en el segundo se ha agregado de manera específica a la infusión de las hojas del cordoncillo.

Las especies Brugmansia suaveolens y Piper sp. han sido usadas más intensamente de manera específica en la pandemia. En el caso de B. suaveolens, la planta ha sido usada tradicionalmente para fortalecer el cuerpo y el espíritu, pero su uso no había sido registrado para tratar enfermedades respiratorias en el pueblo urarina, siendo una posible adaptación para combatir una enfermedad mucho más agresiva. En el caso de Piper sp. ha sido usada para combatir enfermedades respiratorias, pero en menor medida que otras plantas, como la mucura o el ajo sacha. Es probable que su uso específico durante la pandemia haya estado motivado en cierta forma por la publicidad que se le dio al uso de la planta en otras zonas de la Amazonía y la necesidad de contar con armas eficientes contra la enfermedad.

\section{DISCUSIÓN}

En la categoría de plantas usadas para combatir los síntomas del COVID-19, nuestros resultados reportan el uso que la comunidad de Nueva Unión hace de cuatro especies vegetales que no son originarias de América y que fueron introducidas tardíamente en el Perú a partir del siglo XVI (Machuca, 2013). El ajengibre o kión, introducido de manera incipiente por los religiosos españoles y los primeros colonos 
chinos que llegaron a sus costas durante el siglo XVI y popularizado posteriormente por la gran oleada de trabajadores asalariados chinos que llegaron a partir del siglo XIX (Machuca, 2013; Vilches, 2016), tuvo una rápida distribución por todo el país, llegando posiblemente a la Amazonía con las primeras olas extractivas que llegaron atraídas por los recursos naturales de los bosques. La introducción de la toronja y del limón es incierta, pero es muy probable que llegaran con los primeros conquistadores y colonos españoles, teniendo en cuenta que la mayoría de los cítricos ya eran cultivados en Europa desde la Edad Media (Zaragoza, 2007; Zaragoza, 2017). Lo mismo ocurrió posiblemente con la malva, originaria de Europa y muy probablemente introducida en América por las primeras oleadas de colonos que arribaron a sus costas. Todas estas especies fueron adoptadas rápidamente en América y pronto llegarían a las comunidades amazónicas, donde hoy forman parte indiscutible de su botiquín vegetal.

El uso del ajengibre Zingiber officinale para el tratamiento de las afecciones respiratorias ha sido ampliamente descrito en la literatura científica (Tillán et al., 2007; Nepomuceno \& Ishiki, 2010; Terry et al., 2011; Arora et al., 2011; Yachachin, 2013; Carretero, 2015; Villanueva, 2016; Shahrajabian et al., 2019; Mostacero et al., 2020), señalando muchas de estas investigaciones sus propiedades antiinflamatorias, antigripales, antibacterianas, antivirales, analgésicas y expectorantes. Algunas de las investigaciones desarrolladas señalan incluso la eficacia de algunos de los componentes de la raíz de ajengibre frente al virus SARS-CoV-2 (Rathinavel et al., 2020; Rajagopal et al., 2020; Yang et al., 2020, Babatunde et al., 2020). Los usos específicos del ajengibre para combatir la pandemia del COVID-19 han sido reportados por (El Alami et al., 2020), que señalan como la decocción de las raíces de Zingiber y Alpinia officinarum han sido usadas en Marruecos para minimizar los síntomas provocados por el virus. Haridas et al. (2021) señalan el buen potencial que tiene el preparado tradicional elaborado con Citrus medica y Z. officinale para reducir la carga viral e incluso eliminar el SARS-CoV-2 presente en las fosas nasales, uno de las principales puertas de entrada del virus a nuestro organismo. Obeta (2020) reporta el uso del ajengibre para combatir los síntomas del COVID-19 en el estado de Enugu en Nigeria. En sus resultados el autor señala que puede ser ingerido solo o en combinación con otras especies vegetales. Sirén et al. (2021) reportan el uso del ajengibre para combatir el COVID-19 en comunidades kichwa de la Amazonía ecuatoriana. En la revisión bibliográfica realizada por Yang et al. (2020) señalan a Z. officinale como una de las plantas alimenticias medicinales que podrían fortalecer el sistema inmunológico frente a la infección por COVID-19, teniendo comprobados efectos favorables frente a las co-infecciones más comunes que la acompañan, reforzando y dando coherencia al uso que la comunidad de Nueva Unión ha realizado de esta especie.

Los cítricos han sido usados tradicionalmente, como fuente importante de Vitamina C, para prevenir y tratar los problemas respiratorios. Algunas recientes investigaciones señalan a la Vitamina $\mathrm{C}$ o ácido ascórbico como un agente antioxidante, antiinflamatorio y antimodulador, $\mathrm{y}$, por lo tanto, como un atractivo candidato terapéutico en la reducción de la carga viral provocada por COVID-19 y el tratamiento de problemas respiratorios graves como la neumonía (Holford et al., 2020; Cuba et al., 2009). La toronja Citrus sp., contiene polifenoles con propiedades antioxidantes que pueden evitar la replicación viral (De la Rosa et al., 2016). Por su parte, el limón Citrus sp., contiene flavonoides como la hesperidina que podrían tener una potencial reacción antiviral (Bellavite \& Donzelli, 2020; Espinosa et al., 2020). Estos datos dan 
coherencia al uso de cítricos como la toronja y el limón en la comunidad de Nueva Unión, siendo una evidencia más de la capacidad que tiene la población indígena de adoptar especies y apropiarse de usos foráneos para enfrentar nuevas amenazas a su salud.

La malva Malachra alceifolia ha tenido un uso muy extendido durante la pandemia en la comunidad de Nueva Unión, siendo usada antes de la pandemia como antipíretico y analgésico en toda la Amazonía peruana; no hemos encontrado reportes de uso durante la pandemia en otras comunidades indígenas amazónicas. No obstante, como ya hemos mencionado, si existen reportes sobre su uso en comunidades amazónicas antes de la pandemia. Huaranca et al. (2013) reportan el uso de la especie como antipirético en la comunidad El Chino, dentro del área de conservación regional Tahmshiyacu-Tahuayo, en la región peruana de Loreto. Vargas (2020) reporta el uso de $M$. alceifolia en la ciudad de Iquitos para combatir la fiebre y el dolor. Rengifo et al. (2020), reportan el uso de M. alceifolia en comunidades del pueblo shawi ubicadas en la provincia de Alto Amazonas en el departamento peruano de Loreto. Los reportes son consistentes con el uso dado en la comunidad de Nueva Unión durante la primera y segunda ola de la pandemia.

Si bien, el algodón no es originario de la Amazonía, su cultivo y uso se remonta a miles de años de antigüedad antes de la llegada de los europeos a las costas de América. Es una especie americana habitual en las comunidades amazónicas. No hemos encontrado registros del uso de las semillas de algodón Gossypium barbadense durante la pandemia en otras comunidades indígenas amazónicas. Las semillas del algodón contienen una sustancia conocida como gosypol que las hace tóxicas, por lo que han sido usadas para tratar la parasitosis intestinal, para las cefaleas, como insecticida, como antimicótico, como antimicrobiano y antifertilidad (Mejía \& Rengifo, 1995; Percy et al., 1996; García, 2008; Delgado, 1999). Es muy probable que gran parte de los componentes tóxicos se volatilicen al tostar las semillas, algo que explicaría su amplio uso como alimento en la Amazonía (Minam, 2014). En la comunidad de estudio son pocos los que tienen plantas de algodón en sus chacras ubicadas en los terrenos de altura. La semilla, por lo general, ha sido usada como elemento de protección de los niños contra la gripe; para ello se confeccionan collares con las semillas o se cuelgan de las pequeñas hamacas que sirven de kuna a los recién nacidos. Es por este motivo que pensamos que el uso de la semilla ha sido un recurso de emergencia para combatir la agresividad de la enfermedad en la comunidad de Nueva Unión.

De las especies nativas amazónicas, la más utilizada en la comunidad de Nueva Unión, sin lugar a dudas, ha sido el ajo sacha Mansoa alliacea. El ajo sacha es probablemente la planta medicinal amazónica más usada y también una de las más estudiadas. La planta tiene propiedades antisépticas, diuréticas, analgésicas, antitusígenas y antipiréticas, siendo usada para el tratamiento de varias enfermedades, como la artritis, el reumatismo, la pneumonía, los resfriados y otras (Tasambay et al., 2017; De La Torre et al., 2008; Granados et al., 2014; Ankita et al., 2015; Sanz et al., 2009). No es de extrañar, por lo tanto, que haya sido usada en la comunidad de Nueva Unión, ya que tradicionalmente ha sido indicada para el tratamiento de la gripe y sus síntomas. Nuestros registros de uso se corresponden con los reportados por (Siren et al., 2020) en comunidades kichwa de la selva ecuatoriana durante la pandemia, donde un buen número de pobladores atribuyeron su recuperación del COVID-19 a plantas como el ajo sacha, el ajengibre y el chuchuwasi. Kunchikui (2020) reporta el uso de Mansoa alliacea para combatir los síntomas de la gripe en comunidades 
awajun del departamento de Amazonas en Perú, uso que es similar al registrado en la comunidad de Nueva Unión. El autor reporta también el uso de la especie Mansoa parvifolia de manera específica para el COVID-19.

El uso de la ishanga Urera sp. para aplacar los dolores, ha sido reportado dentro y fuera de la Amazonía. Zariquiey \& Fleck (2014) señalan como varias especies del género Urera son usadas para disminuir el dolor en las comunidades del pueblo kakataibo en los departamentos peruanos de Ucayali y Huánuco. Ocampo \& Duro (1994) reportan como las comunidades indígenas dentro de la reserva Tayni de Costa Rica golpean las zonas adoloridas del cuerpo con varias especies del género Urera para aplacar el dolor. La Rotta (1988), reporta como los integrantes del pueblo miraña del Amazonas golpean las zonas adoloridas del cuerpo con las hojas de la especie Urera baccifera. Ducke (1975) informa cómo los cuna de Colombia y Panamá se golpean la cabeza con la planta para el dolor de los pies y los pies para el dolor de cabeza. Estos reportes coinciden con el uso dado a la especie por la comunidad de Nueva Unión y dan consistencia a nuestros resultados. El uso de Urera sp. es tradicional y ha sido un recurso muy utilizado para combatir los dolores de espalda y extremidades provocados por la fiebre.

Los síntomas provocados por el COVID-19 son, en ocasiones, similares a los provocados por una gripe o un resfriado. El uso que la comunidad de Nueva Unión ha dado a la lancetilla Alternanthera brasiliana reside en la eficacia de los preparados elaborados con esta planta para tratar los síntomas gripales y la fiebre. Los usos de esta especie para estos fines son reportados frecuentemente en la literatura científica. Fontana (2007) reporta el uso de la especie como antigripal en comunidades de los estados brasileños de Santa Catarina y Rio Grande do Sul. Agra et al. (2007) informa sobre los usos de $A$. brasiliana para combatir la gripe y los catarros en comunidades ubicadas en el noreste de Brasil; en este reporte el uso de la inflorescencia en infusión está indicado para combatir la gripe y los catarros, mientras que la infusión de las hojas está indicada para reducir la fiebre. En ambos estudios la vía de administración es oral, a través de infusiones, algo que no coincide con nuestros resultados de uso que incorporan la vía tópica a través de baños corporales con la infusión de las hojas. En el estudio de Agra et al. (2007) las partes de la planta usadas para combatir la gripe y los catarros (inflorescencias) difieren de las reportadas en nuestros resultados (hojas). Bussmann \& Sharon (2015) reportan el uso de la planta entera para combatir la tos y la bronquitis; la planta se hierve en agua y se ingiere como agua del tiempo. Estos autores informan sobre el uso de la planta en baños tópicos para combatir los sustos de altura, vía tópica que coincide con nuestros resultados, pero con indicación diferente a la encontrada en Nueva Unión. De la misma forma, Vega (2001) señala el uso que se le da a las hojas en infusión para tratar la fiebre y la bronquitis, pero no reportan un uso tópico de la infusión o el macerado de la hoja.

El uso del cordoncillo o matico Piper sp. en la Amazonía ha sido ampliamente informado en medios no científicos durante la pandemia, no obstante, ya era usado ampliamente para tratar problemas respiratorios en las comunidades indígenas amazónicas. Valadeau et al. (2009) reportan como el pueblo yanesha del Perú usa la infusión de las hojas de varias especies del género Piper para tratar la fiebre y las infecciones en general. Ordinola et al. (2019) reportan el uso que realizan los pueblos awajun y wampis de la región Amazonas del Perú para tratar la fiebre. Rengifo (2007) señala entre alguno de sus usos el tratamiento de la bronquitis y la cicatrización de ulceras, este último uso es consistente con los resultados reportados en nuestro estudio. 
Ruíz \& Mejía (2020) señalan en su revisión de literatura nacional, el potencial antivírico de la especie Piper aduncum, reportando su uso en el Perú para tratar la tos, la fiebre y el resfrío. La vía de administración reportada por estos investigadores coincide con la reportada en nuestros resultados. Los datos proporcionados por nuestros informantes señalan que la planta ha tenido un uso exclusivo para combatir el COVID-19 y que no era muy usada anteriormente, es probable que el uso haya sido favorecido por la publicidad dada a la planta en medios no científicos durante la pandemia.

La uña de gato Uncaria guianensis es otra de las especies cuyo uso ha aumentado considerablemente en la Amazonía durante la pandemia. Algunos estudios recientes señalan el gran potencial que tiene la especie como posible tratamiento frenteal COVID-19 (Yepes etal, 2020). Antes de la pandemia la uña de gato ha sido usada como antiinflamatorio, antioxidante, antiviral y como refuerzo del sistema inmunológico en toda la Amazonía (Rengifo, 2007; Erowele \& Kalejaiye, 2009). La vía de administración más común ha sido la oral, hirviendo la corteza en agua e ingiriendo posteriormente como agua del tiempo. Esta forma de uso coincide con la reportada en el presente estudio.

Son varios los reportes consultados que informan del uso de diferentes especies del género Aspidosperma para combatir la fiebre dentro y fuera de la Amazonía (Perea, 2012; Paéz \& Fonseca, 2014; Giraldo, 2000; Carrizo et al, 2002). El uso en todos los reportes consultados coincide con el reportado por nuestro equipo de investigación para el remo caspi Aspidosperma sp. en la comunidad de Nueva Unión.

La hoja de guayaba Psidium guajava contiene quercetina, un compuesto antiespasmódico que retrasa el movimiento intestinal y retrasa el vaciado gástrico; el uso de su tintura disminuye la producción de toxinas de Escherichia colli y cólera, teniendo efecto antidiarreico comprobado (Rodríguez et al., 2013, Echemendía, 2004). Es por este motivo que es usada ampliamente dentro y fuera de la Amazonía (Desmarchelier et al., 1996; Artero et al., 2015; Kamath et al., 2008; Joseph \& Priya, 2011; Dakappa et al., 2013). El uso de las hojas en infusiones es común en todos los reportes de uso sobre la especie, lo que da consistencia a nuestros resultados.

Otro de los resultados importantes de la investigación ha sido la confirmación del uso que la comunidad de Nueva Unión sigue realizando de ciertas plantas que fortalecen el espíritu frente a posibles amenazas externas y que caracterizan las técnicas terapéuticas propias del chamanismo urarina. Por ejemplo, la función que cumple la ingesta de los macerados a base de ayahuasca, toé y tabaco en el proceso de aprendizaje chamánico es un paso fundamental en la formación de los futuros ayahuasqueros. A través de las ceremonias de ingesta, requisito indispensable para la adquisición de técnicas terapéuticas avanzadas, se produce un cambio en el neófito: sus canales perceptivos se abren, permitiéndole entrar en comunicación con el mundo no humano e interactuar libremente con distintas entidades. De esta forma, el futuro ayahuasquero adquiere no solamente la capacidad de aumentar la movilidad natural de su espíritu, sino también propicia el fortalecimiento del vínculo que su espíritu guarda con el cuerpo, proporcionando una mejor y más duradera protección de las enfermedades, clásicamente atribuidas a causas endógenas, como en el caso de los ataques originados por la voluntad de espíritus dañinos. Sin embargo, estas habilidades no son adquiridas una vez por todas, sino que requieren de una dedicación constante que implica la asunción periódica de los macerados psicótropos, proscripciones alimentarias, abstinencia sexual y el ejercicio constante de las principales técnicas terapéuticas. El COVID-19 ha 
sido una de las amenazas más importantes desde que las últimas epidemias como el sarampión, la viruela y el cólera, golpearan de manera muy fuerte las comunidades urarina a mediados del siglo XX. De igual manera a como hicieron en el pasado, hoy en día las comunidades indígenas han recurrido a las plantas que tradicionalmente fueron utilizadas para fortalecer el cuerpo y el espíritu, los ayahuasqueros urarina han asumido, en muchos casos la primera línea de batalla contra el virus.

El uso de ciertas especies vegetales para fortalecer el cuerpo y el espíritu o para mejorar o amplificar ciertas destrezas como la caza o la pesca ha sido descrito ampliamente en la literatura científica (Tournon \& Silva, 1988; Tournon, 1991; Shepard, 2003). Para muchos pueblos indígenas, las propiedades medicinales o mágicas que tienen ciertas plantas son determinadas por la esencia vital o alma que confiere vida a todo lo que existe. El alma, un concepto que no debe ser confundido con el alma de la tradición occidental, es la que infunde las propiedades que presentan las plantas y que nosotros podemos conocer de manera sensorial. Por ejemplo, cuando una planta es cocinada en agua, "contamina" la infusión con su alma y la persona que la ingiere asimila las propiedades de esa planta (Daly \& Shepard, 2019).

El uso que la comunidad de Nueva Unión hace de las plantas identificadas para fortalecer el espíritu no debe ser interpretado desde el plano biológico/fisiológico, ya que estas plantas no generan una inmunidad frente a patógenos biológicos, sino más bien favorecen la transferencia de ciertas propiedades de estas plantas que permiten ahuyentar, alejar o enfrentar estas amenazas.

Los usos de las plantas conocidas localmente como piri piri Cyperus sp. en la Amazonía han sido registrados en numerosos documentos históricos e investigaciones (Veigl, 1768; Bennet,
1992; Tournon et al., 1998). Bennet (1992) señala como algunas especies de Cyperus son usadas en la Amazonía para inducir el parto, regular la menstruación, eliminar los espíritus malignos y tratar otras enfermedades. Fericgla (2000) informa sobre el uso de ciertas especies de Cyperus para mejorar la destreza de los hombres del pueblo indígena achuar en las actividades de caza. Bourdy et al. (2008) señalan como el pueblo yanesha usa los bulbos de varias especies de Cyperus para protegerse de las enfermedades. Olawsky (2006) señala en su investigación sobre la gramática del pueblo urarina, como algunos tipos de piri piri (biri) son usados por el pueblo urarina por sus poderes mágicos, pero no señala el uso específico de estas especies para proteger al espíritu o al cuerpo de las enfermedades. Nuestra investigación coincide con todas las mencionadas en el registro del uso de la papa o bulbo rallado o molido para protegerse de las enfermedades y en este caso del COVID-19.

La toma de la ayahuasca Banisteriopsis sp. y el toé Brugmansia suaveolens con fines medicinales o chamánicos en la Amazonía ha sido también ampliamente informada (Bennett, 1992; Viegas \& Berlanda, 2012). Hurtado (2000) señala como el uso de la ayahuasca en el Perú permite al ayahuasquero formular una hipótesis etiológica y tomar control de la enfermedad. Bennet (1992) señala como la ayahuasca y el toé son las plantas alucinógenas más importantes del pueblo shuar; los shamanes shuar dialogan con los espíritus bajo la influencia de la ayahuasca; los jóvenes shuar beben el jugo del toé para tener la visión espiritual o arutam. Martin et al. (2019) señalan como los ayahuasqueros urarina ingieren el preparado hecho con la ayahuasca para contactar con las personas que han sido secuestradas por el baiinu, un espíritu del bosque que vive en los aguajales (Schulz et al., 2019).

La ayahuasca, el toé y el tabaco son las plantas chamánicas psicoactivas más importantes para 
el pueblo urarina, debido a que su uso se vincula a distintas prácticas chamánicas y terapeúticas (Dean, 2009; Fabiano \& Nuribe, 2021). El toé ha sido una planta mixta, ya que además de ser utilizada para fortalecer el cuerpo y una de las dos componentes anímicas de la persona identificada con el nombre de kurii (el espíritu), ha sido también utilizada como antipirético y analgésico. El toé tiene como uno de sus componentes la escopolamina, un alcaloide que inhibe ciertas funciones neurológicas que tienen que ver con la sensación del dolor. Es por este motivo que ha sido usada para mitigar los dolores provocados por el COVID-19. Esta duplicidad también ha sido reportada con Brugmansia aurea en comunidades del valle del Sibundoy, en el Alto Putumayo, Colombia (Rodríguez, 2010).

Son los ayahuasqueros urarina (jaera) los encargados de elaborar los preparados con estas plantas, de activarlos y de volverlos inofensivos y eficaces a través del uso de cantos del género aaü (Fabiano, 2014). La toma del preparado a base de ayahuasca o toé, acompañada de cantos de activación y de cantos de protección ha sido una tónica durante la pandemia en la comunidad de Nueva Unión. Muchos pobladores han ingerido los preparados para hacer frente al coronavirus, bajo la supervisión de los pocos ayahuasqueros que aún quedan en ella, fortaleciendo de esta forma el cuerpo y el espíritu, desempeñando por lo tanto una función profiláctica y evitando la presentación de los síntomas cuando viajaban a la ciudad o a otras comunidades.

En lo que respecta a la mucura Petiveria alliacea, también es una planta muy usada en toda la región amazónica, tanto para combatir la fiebre y la gripe, como para fortalecer el cuerpo y el espíritu. Específicamente, el uso de la planta como protectora del cuerpo en la Amazonía peruana, ha sido informado por Jaúregui et al. (2011) que reportan como las comunidades indígenas de la selva central del Perú usan sus hojas en forma de baños protectores contra las energías negativas. Tudela \& La Torre (2015) señalan cómo el pueblo shipibo konibo la considera una planta madre y la usa para tratar la fiebre, la gripe, la diarrea, el reumatismo y la picadura de la hormiga isula Paraponera clavata, pero también como protección contra la brujería, la mala suerte y para mejorar la habilidad cinegética, referencia que se ajusta a los usos reportados en nuestra investigación en la comunidad de Nueva Unión. Rondina et al. (2008) reporta la inhalación del humo de la raíz de $P$. alliacea para controlar el dolor de cabeza en comunidades de la república Argentina, estos autores no reportan un uso protector para el cuerpo y el espíritu. La presencia de esta planta en las comunidades urarina ubicadas en el río Corrientes ha sido reportada por Perrault \& Coomes (2008), quienes la califican como una planta ocasional en la agrobiodiversidad de la zona; en la comunidad de Nueva Unión las familias cuentan con esta planta en sus chacras familiares, siendo, como ya mencionamos antes, muy utilizada en la comunidad, algo que no concuerda con los resultados de estos investigadores, difiriendo claramente de los nuestros.

\section{CONCLUSIONES}

La pandemia ha sido un gran desafío para las comunidades indígenas amazónicas en el Perú. Los resultados de nuestra investigación corroboran la vigencia del conocimiento tradicional sobre el uso de las plantas medicinales y chamánicas en la comunidad urarina de Nueva Unión. Las 16 especies vegetales identificadas han sido las únicas armas con las que ha contado esta comunidad para enfrentar la pandemia. Debido a la lejanía de los centros de salud y a la escasa atención del estado, sobre todo durante la primera ola de contagios, la comunidad tuvo que 
echar mano de los conocimientos tradicionales sobre las plantas que curan y hacer frente a una de sus mayores amenazas durante el presente siglo. Los resultados evidencian también como la comunidad ha adaptado los usos tradicionales a la nueva realidad y ha adoptado ciertos preparados como nuevas armas para enfrentar una enfermedad desconocida y muy agresiva. Entre el arsenal medicinal contra el virus encontramos cuatro especies que no son originarias de América pero que han sido integradas a las prácticas medicinales de la comunidad. Se evidencia la importancia de cuatro especies vegetales que han sido usadas como fortalecedoras del cuerpo y el espíritu; dos de ellas han sido usadas también para combatir algunos síntomas de la enfermedad. El bajo porcentaje de letalidad en la comunidad de Nueva Unión durante la pandemia $(<1 \%)$, a pesar del contagio generalizado de sus pobladores, podría ser un indicador de la eficacia anti COVID-19 de algunos preparados usados en la comunidad. Las investigaciones reportadas sobre algunas de las especies usadas son todavía muy incipientes, pero hay indicios de que algunas pueden tener propiedades antivirales que podrían ser efectivas frente al COVID-19, por lo que se recomienda desarrollar más investigaciones sobre los compuestos contenidos en las especies $\mathrm{y}$ sus potenciales aplicaciones para combatir enfermedades respiratorias provocadas por virus o bacterias.

\section{AGRADECIMIENTOS}

A la comunidad de Nueva Unión, a sus mujeres y hombres, por haber compartido sus conocimientos en una época de pandemia muy complicada y dramática, por su fortaleza y férrea voluntad de conservar los conocimientos tradicionales del pueblo urarina.

\section{REFERENCIAS BIBLIOGRÁFICAS}

Agra, M.; Freita, P.; Barbosa, J.M. 2007. Synopsis of the plants known as medicinal and poisonous in Northeast of Brazil. Revista Brasileira de Farmacognosia. 17 (1): 114140. DOI: https://doi.org/10.1590/S0102695X2007000100021

Ankita, S.; Chandra, S.; Arti, T. 2015. Free radical scavenging activity of leaves of Adenocalymma alliaceum. European Journal of Biomedical and Pharmaceutical Sciences, 2(3): 1035-1038.

Arora, R.; Chawla, R.; Marwah, R.; Arora, P.; Sharma, R.K.; Kaushik, V.; Goel, R.; Kaur, A.; Silambarasan, M.; Tripathi, R.P.; Bhardwaj, J.R. 2011. Potential of complementary and alternative medicine in preventive management of novel H1N1 flu (swine flu) pandemic: Thwarting potential disasters in the bud. Evidence-Based Complementary and Alternative Medicine, 2011: 586506. DOI: https://doi.org/10.1155/2011/586506

Artero, G. J. 2015. Elaboración de jugo funcional de guayaba (Psidium guajava L.) a partir de mermas y coproductos de la producción de guayaba en El Salvador. Tesis de pregrado. Escuela Agrícola Panamericana, Zamorano Honduras. 42pp.

Babatunde, J.; Akinwunmi, A.; Olaoye, I. 2020. Pharmacoinformatics and hypothetical studies on allicin, curcumin, and gingerol as potential candidates against COVID-19-associated proteases. Journal of Biomolecular Structure and Dynamics, DOI: https://doi.org/10.1080/ 07391102.2020.1813630

Bellavite, P.; Donzelli, A. 2020. Hesperidin and SARS-CoV-2: New light on the healthy function of citrus fruits. Antioxidants (Basel, Switzerland), 9(8): 742. DOI: https://dx.doi. org/10.3390\%2Fantiox9080742

Bennett, B.C. 1992. Hallucinogenic plants of the shuar and related indigenous groups in 
amazonian Ecuador and Peru. Brittonia, 44(4), 483. DOI: https://doi.org/10.2307/2807199

Bourdy, G.; Valadeau, G.; Albán, J. 2008. Yato'ramuesh: plantas medicinales yaneshas. IRD Editions, Marsella. 345pp.

Bussmann, R.; Sharon, D. 2015. Plantas medicinales de los Andes y la Amazonia. La flora mágica y medicinal del norte del Perú. Jardín Botánico de Missouri, Trujillo. 292pp.

Carretero, M.E. 2015. Gengibre (Zingiber officinale): un posible agente antiinflamatorio. Panorama Actual del Medicamento, 39(382): 330-333

Carrizo, E.; Palacio, M.; Roic, L. 2002. Plantas de uso medicinal en la flora de los alrededores de la ciudad de Santiago del Estero. Dominguezia, 18: 26-35.

Cordero, M. 2001. Las grandes epidemias en la América colonial. Archivos de Zootecnia. 50: 597-612.

Crosby, A. 1972. The Columbian exchange: biological and cultural consequences of 1492. Duke University Press, Westport, Conn. 277pp.

Cuba, M.; Lacasse, L.R; Mariño, O.; Gómez, E. 2009. Ventajas de la frutoterapia en pacientes convalecientes de neumonías graves. Revista Cubana de Enfermería, 25(1-2): 1-10.

Dakappa, S.; Roshan, A.; Sharma, S.; Sunita, S. 2013. A review on the medicinal plant Psidium guajava Linn. (Myrtaceae). Journal of Drug Delivery \& Therapeutics, 3(2): 162-168. DOI: https://doi.org/10.22270/jddt.v3i2.404

Daly, L.; Shepard, G.H. 2019. Magic darts and messenger molecules: a sensory ecology of human-plant engagements in Amazonia. Anthropology Today, 35(2): 13-17. DOI: https://doi.org/10.1111/1467-8322.12494

Dean, B. 2009. Urarina society, cosmology, and history in peruvian Amazonia. University Press of Florida, Gainesville. 315pp.

De la Rosa, M.; Wong, J.; Muñiz, D.; Carrillo, M.; Sánchez, J. 2016. Compuestos fenólicos bioactivos de la toronja (Citrus paradisi) y su importancia en la industria farmacéutica y alimentaria. Revista Mexicana de Ciencias Farmacéuticas, 47(2): 22-35.

De La Torre, L.; Navarrete, H.; Muriel, M. P.; Macía, M. J.; Balslev, H. (eds.) 2008. Enciclopedia de las plantas útiles del Ecuador. Herbario QCA de la Escuela de Ciencias Biológicas de la Pontificia Universidad Católica del Ecuador, Quito. 949pp.

Delgado, H. 1999. Inventario de recursos curativos en centros de expendio formales e informales: Puerto Maldonado. Instituto Nacional de Medicina Tradicional, Lima. 25pp.

Denevan, W. 1992. The native population of the Americas in 1492. University of Wisconsin Press, Madison. 353pp.

Desmarchelier, C.; Gurni, A.; Ciccia, G.; Giuletti, A. M. 1996. Ritual and medicinal plants of the Ese'ejas of the Amazonian rainforest (Madre de Dios, Perú). Journal of Ethnopharmacology, 52(1): 45-51. DOI: https://doi. org/10.1016/0378-8741(96)01390-6

Ducke, J. 1975. Ethnobotanical observations on the Cuna Indians. Economic Botany, 29(3): 278-293. DOI: https://doi.org/10.1007/ bf02873178

Echemendía, C.; Morón, F. 2004. Tintura de hojas de Psidium guajava L. en pacientes con diarrea aguda simple. Revista Cubana de Plantas Medicinales, 9 (3): 479620040003.

El Alami, M. A.; Fattah, A.; Chait, A. 2020. Medicinal plants used for the prevention purposes during the COVID-19 pandemic in Morocco. Journal of Analytical Sciences and Applied Biotechnology, 2(1): 4-11. DOI: https://doi.org/10.48402/ IMIST.PRSM/jasab-v2i1.21056

Erowele, G. I; Kalejaiye, A.0. 2009. Pharmacology and therapeutic uses of cat's claw. American Journal of Health-System Pharmacy, 66(11): 992-995. DOI: https://doi.org/10.2146/ ajhp080443 
Espinosa, S.; Asaf, A.; Salinas, V. 2020. Medicina alternativa para infecciones respiratorias de etiología viral. Revista RD ICUAP, 19(7): 26-46.

Fabiano, E. 2014. Función terapéutica de los cantos Urarina. En: Universidad de Ayacucho Federico Froebel. Descubriendo nuestras identidades. I Congreso Latinoamericano de Etnopsicología. p.137-150.

Fabiano, E.; Nuribe, S. 2021. Kurunabirus: una mirada urarina sobre la creación de las enfermedades y los nuevos contagios en la cuenca del río Chambira (Amazonía peruana). Mundo Amazónico, 12(1): 187-200. DOI: https://doi.org/10.15446/ma.v12n1.88921

Fericgla, J. M. 2000. Los jibaros cazadores de sueños. Editorial Abya Yala, Quito. 382pp.

Fontana, D. 2007. Morfoanatomía e histoquímica comparativa entre Alternanthera brasiliana (L.) Kuntze e Alternanthera dentata (Moench) Stuchlik; estudo fitoquímico e biológico de Alternanthera brasiliana. Tesis de maestría. Universidade Federal de Santa María, RS. $111 p p$.

García, F. 2008. Impacto antrópico en las plantas medicinales nativas del departamento de Amazonas, Perú. Tesis de doctorado. Universidad Nacional de Trujillo. 110pp.

Giraldo, C. 2020. Medicina tradicional de las mujeres inga. Revista de la Academia Colombiana de Ciencias Exactas, Fisicas y Naturales, 24(90): 5-23.

Granados, C.; Pérez, R.; Soto, M.; Ruiz, J.; Lagunez, L.; Alonso, N.; Gato, R. 2014. Inhibition of the growth and development of mosquito larvae of Culex quinquefasciatus (Diptera: Culicidae) treated with extract from leaves of Pseudocalymma alliaceum (Bignonaceae). Asian Pacific Journal of Tropical Medicine, 7(8): 594-601. DOI: https://doi.org/10.1016/ s1995-7645(14)60101-2

Haridas, M.; Sasidhar, V.; Nath, P.; Abhithaj, J.; Sabu, A.; Rammanohar, P. 2021. Compounds of Citrus medica and Zingiber officinale for COVID-19 inhibition: in silico evidence for cues from Ayurveda. Future Journal of Pharmaceutical Sciences, 7(1): 13. DOI: https://dx.doi.org/10. 1186\%2Fs43094-020-00171-6

Holford, P.; Carr, A.; Jovic, T.; Ali, S.; Whitaker, L.; Marik, P.; Smith, D. 2020. Vitamin C, an adjunctive therapy for respiratory infection, sepsis and COVID-19. Nutrients, 12: 3760. DOI: https://doi.org/10.3390/nu12123760

Huaranca, R.; Armas, J.; Vigo, R. 2013. Uso de las plantas medicinales en la comunidad El Chino, del área de conservación regional comunal Tamshiyacu-Tahuayo, Loreto, Perú. Conocimiento Amazónico, 4(2): 77-86.

Hurtado, L. 2000. Perfil cultural del shamanismo de la Amazonía peruana. En: Alva, W.; Polia, M; Chávez, F.; Huratado, L. Shamán: la Búsqueda. Imprenta San Pablo, Córdoba. p. 225-69.

Instituto Nacional de Estadística. 2017. Censos Nacionales. XII de Población, VII de Vivienda y III de Comunidades Indígenas. INEI, Lima. 644pp.

Jaúregui, X.; Clavo, Z.M.; Jovel, E.M.; Pardo, M. 2011. Plantas con madre: plants that teach and guide in the shamanic initiation process in the east-central peruvian Amazon. Journal of Ethnopharmacology, 134(3): 739-752. DOI: https://doi.org/10.1016/j.jep.2011.01.042

Joseph, B.; Priya, M. 2011. Review on nutritional, medicinal and pharmacological properties of Guava (Psidium guajava Linn.). International Journal of Pharma and Bio Sciences, 2(1): 5369.

Judd, W.; Campbell, C.; Kellogg, E.; Stevens, P. 1999. Plant systematics: a phylogenetic approach. Sinauer, Sunderland - UK. 464pp.

Kamath, J.V.; Rahul, N.; Ashok, C.V; Lakshmi, M.; Devi, S. 2008. Psidium guajava L.: a review. International Journal of Green Pharmacy, 2(1): 9-12. DOI: http://dx.doi.org/10.22377/ijgp. v2i1.386 
Kunchikui, J. 2020. Evaluación etnobotánica en la comunidad nativa Numpatkaim, distrito Imaza, Provincia Bagua, Amazonas. Tesis de pregrado. Universidad Nacional de Cajamarca. 128pp.

La Rotta, C. 1989. Especies utilizadas por la comunidad Miraña (Estudio etnobotánico). World Wildlife Fund, Bogotá. 386pp.

Ludescher, M. 2001. Instituciones y prácticas coloniales en la Amazonía peruana: pasado y presente. Indiana, 17-18: 313-359. DOI: https://doi.org/10.22490/24629448.1006

Mejía, K.; Rengifo, E. 1995. Plantas medicinales de uso popular en la Amazonía peruana. Instituto de Investigaciones de la Amazonía Peruana (IIAP), Iquitos. 285pp.

MINAM. 2014. Colecta, elaboración de mapas de distribución y estudio socioeconómico de la diversidad del algodón nativo. Informe final. Ministerio del Ambiente, Lima. 83pp.

MINSA. 2021. Sala situacional COVID-19 Perú. (https://covid19.minsa.gob.pe/sala_ situacional.asp). Acceso: 18/03/2021.

Mostacero, J.; López, S.; De la Cruz, A.; GilRivero, A.; Alva-Calderón, R.; Charcape, M. 2020. Plantas frías y plantas calientes, recursos potenciales en la prevención y/o tratamiento del COVID-19. Manglar 17(3): 209-220. DOI: http://dx.doi.org/10.17268/ manglar.2020.031

Nepomuceno, A.E; Ishiki, M. 2010. Las plantas empleadas para el tratamiento de las infecciones respiratorias en los altos de Chiapas (México). Etnobiología 8(1): 11-30.

Obeta, M.U. 2020. Anti-COVID-19 properties of ginger (Zingiber officinale) assisted Enugu nigerian people during the pandemic. Journal of Bacteriology and Infectious Diseases, 4(3): 5. Ocampo, R.; Duro, J. 1994. Estudio etnobotánico y características socioeconómicas de la comunidad indígena de Tayní, Costa Rica. CATIE, San José - Costa Rica. 25pp.
Olawsky, K. 2006. A grammar of urarina. Mouton de Gruyter, Berlín, Alemania. 943pp.

Ordinola, C.; Barrena, M.A.; Rascón, J.; Corroto, F.; Barrena C.M; Cucho, M.; Mejía, F. 2019. Uso de plantas medicinales para el síndrome febril por los pobladores del Asentamiento Humano Pedro Castro Alva del distrito de Chachapoyas (Chachapoyas - Perú). Arnaldoa, 26(3): 10331046. DOI: http://dx.doi.org/10.22497/ arnaldoa.263.26312

Percy, R.G.; Calhoun, M. C.; Kim, H.L. 1996. Seed Gossypol variation within Gossypium barbadense L. cotton. Crop Science, 36(1): 193-197. DOI: https://doi.org/10.2135/ cropsci1996.0011183X003600010034x

Perea, R.M. 2012. Evaluación de la actividad antimalárica in vitro de extractos de especies vegetales de la familia Apocynaceae de la Amazonia peruana por citometría de flujo. Tesis de pregrado. Universidad Nacional de la Amazonía Peruana, Iquitos. 65pp.

Perrault-Archambault, M.; Coomes, O.T. 2008. Distribution of agrobiodiversity in home gardens along the Corrientes river, peruvian Amazon. Economic Botany, 62(2): 109126. DOI: https://doi.org/10.1007/s12231008-9010-2

Rajagopal, K.; Byran, G.; Jupudi, S.;Vadivelan, R. 2020. Activity of phytochemical constituents of black pepper, ginger, and garlic against coronavirus (COVID-19): An in silico approach. International Journal of Health \& Allied Sciences, 9(5): 43.

Rathinavel, T.; Palanisamy, M.; Palanisamy, S.; Subramanian, A.; Thangaswamy, S. 2020. Phytochemical 6-Gingerol - A promising drug of choice for COVID-19. International Journal Advanced Science Engineering. 6 (4): 14821489.

Rengifo, E. 2007. Las ramas floridas del bosque. Instituto de Investigaciones de la Amazonía Peruana (IIAP), Iquitos. 88pp. 
Rengifo, E.; Fachín, L.; Rodriguez, A.; Palacios, J.J. 2020. El conocimiento tradicional para la mejoría de su salud, con especies medicinales en las comunidades indígenas de la etnia Shawi, en la Amazonia peruana. Medical Plant Communications, 3(3): 45-52. DOI: https:// doi.org/10.37360/mpc.20.3.3.10

Ribeiro, J. E. L. S.; Hopkins, M.J. G.; Vicentini, A; Sothers, C. A.; Costa, M. A. S.; Brito, J. M.; Souza, M.A.D.; Martins, L.H.; Lohmann, L. G.; Assunção, P.A.; Pereira, E. C.; Silva, C. F.; Mesquita, M. R.; Procópio, L. C. 1999. Flora da Reserva Ducke. Guia de identificação das plantas vasculares de uma floresta de terra firme na Amazônia Central. INPA-DFID, Manaus. 800pp.

Rodríguez, J.J. 2010. Uso y manejo tradicional de plantas medicinales y mágicas en el valle del Sibundoy, Alto Putumayo, y su relación con procesos locales de construcción ambiental. Revista de la Academia Colombiana de Ciencias, 34(132): 309-326.

Rodríguez, R.; Lafourcade, A.; Pérez, L. 2013. Hojas de Psidium guajava L. Revista Cubana de Farmacia, 47(1): 127-135.

Rondina, R.; Bandoni, A.; Coussio, J. 2008. Especies medicinales argentinas con potencial actividad analgésica. Dominguezia, 24(1): 4769.

Ruíz, P.; Mejía, F. 2020. Plantas utilizadas en medicina tradicional para afecciones respiratorias virales. Revista de Investigación Científica REBIOL, 40(1): 109-130.

Sanz, J.; Campos, J.; Epiquién, M.A.; Cañigueral, S. 2009. A first survey on the medicinal plants of the Chazuta valley (Peruvian Amazon). Journal of Ethnopharmacology, 122(2): 333-362. DOI: https://doi.org/10.1016/j.jep.2008.12.009

Shahrajabian, M. H.; Sun, W.; Cheng, Q. 2019. Clinical aspects and health benefits of ginger (Zingiber officinale) in both traditional Chinese medicine and modern industry. Acta Agriculturae Scandinavica, Section B - Soil \& Plant Science, 69(6): 546-556. DOI: https:// doi.org/10.1080/09064710.2019.1606930

Shepard, G.H.; Izquierdo, C. 2003. Los Matsiguenka de Madre de Dios y del Parque Nacional del Manu. En: B. Huertas; A. Garcia (Eds.), Los pueblos indígenas de Madre de Dios: Historia, etnografía e coyuntura. IWGIA, p. 111-126.

Schulz, C.; Martín, M.; Del Águila, M.; Laurie, N.; Lawson, I.; Núñez, C.; Roucoux, K. 2019. Peatland and wetland ecosystems in Peruvian Amazonia: indigenous classifications and perspectives. Ecology and Society, 24(2): 12. DOI: https://doi.org/10.5751/ES-10886240212

Siren, A.; Uzendoski, M.; Swanson, T.; Jacome, I.; Siren, E.; Tapia, A.; Dahua, A.; Tanguila, A.; Santi, E.; Machoa, D.; Andi, D.; Santi, D. 2020. Resiliencia contra la pandemia de COVID-19 en comunidades indígenas kichwa en la Amazonía ecuatoriana. Revista Latinoamericana de Políticas y Acción Pública, 7(2): 101-107. DOI: https://doi. org/10.17141/mundosplurales.2.2020.4738

Tasambay, A.; Scalvenzy, L.; Piedra, A.S.; Radice, M. 2017. Ethnopharmacology, biological activity and chemical characterization of Mansoa alliacea. A review about a promising plant from Amazonian region. Mold2net, 3(2017): 04617. DOI: https://doi.org/10.3390/ mol2net-03-04590

Terry, R.; Posadzki, P.; Watson, L.; Ernst, E. 2011. The use of ginger (Zingiber officinale) for the treatment of pain: a systematic review of clinical trials. Pain Medicine, 12(12): 18081818. DOI: https://doi.org/10.1111/j.15264637.2011.01261.x

Tillán, J.; Núñez, Y.; Agüero, S.; Carrillo, C. 2007. Actividad antiinflamatoria de compuestos liposolubles de Zingiber officinale Roscoe frente a diferentes agentes flogísticos. Revista Cubana de Plantas Medicinales, 12(2).

Tournon, J.; Silva, M. 1988. Plantas para cambiar el comportamiento humano entre los ShipiboConibo. Anthropologica, 6(6): 161-176. 
Tournon, J. 1991. La clasificación de los vegetales entre los Shipibo-Conibo. Anthropologica, 9(9): 121-151.

Tudela, P.; La Torre, M. 2015. Cultural importance and use of medicinal plants in the ShipiboConibo native community of Vencedor (Loreto) Peru. Ethnobotany Research and Applications, 14: 533-548.

Valadeau, C.; Pabón, A.; Deharo, E.; Albán, J.; Estévez, Y.; Lores, F.; Rojas, R.; Gamboa, D.; Sauvain, M.; Castillo, D.; Bourdy, G. 2009. Medicinal plants from the Yanesha (Peru): Evaluation of the leishmanicidal and antimalarial activity of selected extracts.Journal of Ethnopharmacology, 123(3): 413-422. DOI: https://doi.org/10.1016/j.jep.2009.03.041

Vargas, G. 2020. Plantas medicinales con valor comercial en la ciudad de Iquitos, Perú. Medicinal Plant Communications, 3(1): 12-16. DOI: https://doi.org/10.37360/mpc.20.3.1.3

Vásquez, R. 1997. Flórula de las Reservas Biológicas de Iquitos, Perú, Allpahuayo- Mishana, Explornapo Camp, Explorama Lodge. Missouri Botanical Garden, St. Louis, MO (Monographs in Systematic Botany, 63). 1046pp.

Vega, M. 2001. Etnobotanica de la Amazonia Peruana. Ediciones Abya Yala, Quito. 166pp.

Veigl, F.C. [1798]. Noticias detalladas sobre el estado de la provincia de Maynas en América Meridional hasta el año de 1768. CETA, Iquitos, Perú. 261pp.

Vilches, H. 2016. Hacia una nueva diversidad: Migraciones asiáticas en América Latina. Tiempo y Espacio, 26(65): 99-119.

Villanueva, M. 2016. Efecto antibacteriano del extracto acuoso del Zingiber officinale "kión", sobre cepas de Staphylococus aureus comparado con gentamicina in vitro. Tesis de pregrado. Universidad Cesar Vallejo, Lima. 49pp.

Yachachin, S.L. 2013. Caracterización fisicoquímica del extracto expectorante de ajo (Allium sativum L.), kión (Zingiber officinale L.), eucalipto (Eucaliptus globulus L.) Y linaza (Linum usitatissimum L.). Tesis de pregrado. Universidad Nacional del Centro del Perú, Huancayo. 175pp.

Yang, F.; Zhang, Y.; Tariq, A.; Jiang, X.; Ahamd, Z.; Zhihao, Z.; Idrees, M.; Azizullah, A.; Adnan, M.; Bussmann, R. 2020. Food as medicine: a possible preventive measure against coronavirus disease (COVID-19). Phytotherapy Research, 34(12): 3124-3136. DOI: https://doi. org/10.1002/ptr.6770

Yepes, A.; Herrera, 0.; Sánchez, J.; Tiessler, L.; Didier, J.; Cardona, W. 2020. Investigating potential inhibitory effect of Uncaria tomentosa (cat's claw) against the main protease 3CLpro of SARS-CoV-2 by molecular modeling. EvidenceBased Complementary and Alternative Medicine, 2020: 4932572. DOI: https://doi. org/10.1155/2020/4932572

Zaragoza, S. 2007. Aproximación a la historia de los cítricos. Origen, dispersión y evolución de su uso y cultivo. Tesis doctoral. Universidad Politécnica de Valencia, Valencia. 518pp.

Zaragoza, S. 2017. Los cítricos en Europa durante la edad media. Levante Agrícola: Revista Internacional de Cítricos, 436: 88-92.

Zariquiey, R.; Fleck, D. 2014. Animales y plantas del pueblo kakataibo. Diccionario trilingüe (kakataibo, español, inglés) con idenficaciones biológicas, índice alfabético castellanokakataibo, clasificación semántica, nombres regionales y definiciones etnobiológicas. Lincom, Munich. 266pp.

Recibido: 9 de julio de 2021 Aceptado para publicación: 18 de agosto de 2021 